\title{
Outcome and status of postcardiac arrest care in Korea: results from the Korean Hypothermia Network prospective registry
}

\begin{abstract}
Soo Hyun Kim', Kyu Nam Park², Chun Song Youn², Minjung Kathy Chae ${ }^{3}$, Won Young Kim ${ }^{4}$, Byung Kook Lee ${ }^{5}$, Dong Hoon Lee ${ }^{6}$, Tae Chang Jang ${ }^{7}$, Jae Hoon $\mathrm{Lee}^{8}$, Yoon Hee Choi ${ }^{9}$, Je Sung You ${ }^{10}$, In Soo Choo ${ }^{11}$, Su Jin Kim ${ }^{12}$, Jong-Seok Lee ${ }^{13}$, Yong Hwan Kim ${ }^{14}$, Min Seob Sim ${ }^{15}$, Jonghwan Shin ${ }^{16}$, Yoo Seok Park ${ }^{17}$, Young Hwan Lee ${ }^{18}$, HyungJun Moon ${ }^{19}$, Won Jung Jeong ${ }^{20}$, Joo Suk $\mathrm{Oh}^{21}$, Seung Pill Choi ${ }^{1,22}$, Kyoung-Chul $\mathrm{Cha}^{23}$; on behalf of the Korean Hypothermia Network investigators
\end{abstract}

\begin{abstract}
Department of Emergency Medicine, ${ }^{1}$ Eunpyeong St. Mary's Hospital, The Catholic University of Korea College of Medicine, Seoul; ${ }^{2}$ Seoul St. Mary's Hospital, The Catholic University of Korea College of Medicine, Seoul; ${ }^{3}$ Ajou University Medical Center, Suwon; ${ }^{4}$ Asan Medical Center, University of Ulsan College of Medicine, Seoul; ${ }^{5}$ Chonnam National University Hospital, Chonnam National University Medical School, Gwangju; ${ }^{6}$ Chung-Ang University Hospital, Chung-Ang University College of Medicine, Seoul; ${ }^{7}$ Daegu Catholic University Medical Center, Catholic University of Daegu School of Medicine, Daegu; ${ }^{8}$ Dong-A University Hospital, Dong-A University College of Medicine, Busan; ${ }^{9}$ Ewha Womans University Mokdong Hospital, Ewha Womans School of Medicine, Seoul: ${ }^{10} \mathrm{Gangnam}$ Severance Hospital, Yonsei University College of Medicine, Seoul; ${ }^{11}$ Hanil General Hospital, Seoul; ${ }^{12}$ Korea University Anam Hospital, Korea University College of Medicine, Seoul; ${ }^{13}$ Kyung Hee University Medical Center, Kyung Hee University School of Medicine, Seoul; ${ }^{14}$ Samsung Changwon Hospital, Sungkyunkwan University School of Medicine, Changwon; ${ }^{15}$ Samsung Medical Center, Sungkyunkwan University School of Medicine, Seoul; ${ }^{16}$ Seoul Metropolitan Government Seoul National University Boramae Medical Center, Seoul; ${ }^{17}$ Severance Hospital, Yonsei University College of Medicine, Seoul; ${ }^{18}$ Soonchunhyang University Bucheon Hospital, Bucheon;

${ }^{19}$ Soonchunhyang University Cheonan Hospital, Cheonan; ${ }^{20}$ St. Vincent's Hospital, The Catholic University of Korea College of Medicine, Suwon; ${ }^{21}$ Uijeongbu St. Mary's Hospital, The Catholic University of Korea College of Medicine, Uijeongbu; ${ }^{22}$ Yeouido St. Mary's Hospital, The Catholic University of Korea College of Medicine, Seoul; ${ }^{23}$ Wonju Severance Christian Hospital, Yonsei University College of Medicine, Wonju, Korea
\end{abstract}

Objective High-quality intensive care, including targeted temperature management (TTM) for patients with postcardiac arrest syndrome, is a key element for improving outcomes after outof-hospital cardiac arrest (OHCA). We aimed to assess the status of postcardiac arrest syndrome care, including TTM and 6-month survival with neurologically favorable outcomes, after adult OHCA patients were treated with TM, using data from the Korean Hypothermia Network prospective registry.

Methods We used the Korean Hypothermia Network prospective registry, a web-based multicenter registry that includes data from 22 participating hospitals throughout the Republic of Korea. Adult comatose OHCA survivors treated with TM between October 2015 and December 2018 were included. The primary outcome was neurological outcome at 6 months.

Results Of the 1,354 registered OHCA survivors treated with TTM, 550 (40.6\%) survived 6 months, and 413 (30.5\%) had good neurological outcomes. We identified $839(62.0 \%)$ patients with pre-
elSSN: 2383-4625

Received: 6 April 2020

Revised: 19 June 2020

Accepted: 25 June 2020

Correspondence to: Kyu Nam Park Department of Emergency Medicine, Seoul St. Mary's Hospital, The Catholic University of Korea College of Medicine, 222 Banpo-daero, Seocho-gu, Seoul 06591, Korea

E-mail: emsky@catholic.ac.kr ORCID

https://orcid.org/0000-0001-7108-7690

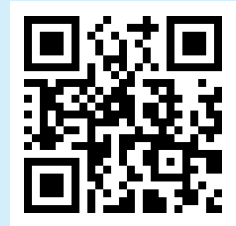

How to cite this article:

Kim SH, Park KN, Youn CS, Chae MK, Kim WY, Lee BK, Lee DH, Jang TC, Lee JH, Choi YH, You JS, Cho IS, Kim SJ, Lee JS, Kim YH, Sim MS, Shin J, Park YS, Lee YH, Moon HJ, Jeong WJ, Oh JS, Choi SP, Cha KC; on behalf of the Korean Hypothermia Network investigators. Outcome and status of postcardiac arrest care in Korea: results from the Korean Hypothermia Network prospective registry. Clin Exp Emerg Med 2020;7(4):250-258. https://doi. org/10.15441/ceem.20.035

This is an Open Access article distributed under the terms of the Creative Commons Attribution Non-Commercial License (https:// creativecommons.org/licenses/by-nc/4.0/). 
sumed cardiac etiology. A total of 937 (69.2\%) collapses were witnessed, shockable rhythms were demonstrated in $482(35.6 \%)$ patients, and 421 (31.1\%) patients arrived at the emergency department with prehospital return of spontaneous circulation. The most common target temperature was $33^{\circ} \mathrm{C}$, and the most common target duration was 24 hours.

Conclusion The survival and good neurologic outcome rates of this prospective registry show great improvements compared with those of an earlier registry. While the optimal target temperature and duration are still unknown, the most common target temperature was $33^{\circ} \mathrm{C}$, and the most common target duration was 24 hours.

Keywords Out-of-hospital cardiac arrest; Hypothermia, induced; Registries; Critical care outcomes

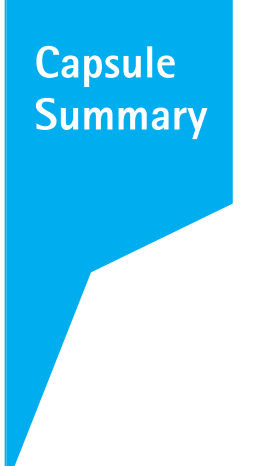

What is already known

High-quality intensive care, including targeted temperature management (TTM) for patients with postcardiac arrest syndrome, is a key element for improving outcomes after out-of-hospital cardiac arrest (OHCA). Several studies have reported the outcome and status of postcardiac arrest care, including $\Pi \mathrm{M}$, in their own countries.

What is new in the current study

Of the 1,354 total out-of-hospital cardiac arrest survivors treated with $\Pi M, 413(30.5 \%)$ had good neurologic outcomes at 6 months. The most common target temperature and duration were $33^{\circ} \mathrm{C}$ and 24 hours. This is the first report from a large-scale multicentered prospective registry in Korea.

\section{INTRODUCTION}

Despite recent advancements in critical care, out-of-hospital cardiac arrest (OHCA) is still one of the major causes of death in many countries. ${ }^{1-3}$ High-quality intensive care, including targeted temperature management (TTM) for patients with postcardiac arrest syndrome (PCAS), is a key element for improving outcomes after OHCA. ${ }^{1,45}$ TM is known to improve survival and neurologic outcomes after $\mathrm{OHCA}^{6-9}$ and several other postcardiac arrest treatment approaches such as emergency coronary angiography (CAG), hemodynamic optimization, ventilator management, and prognostication may also impact patient outcomes. ${ }^{10-19}$

Cardiac arrest etiology and quality of postcardiac arrest care, including $\Pi \mathrm{TM}$, as well as outcomes after OHCA differ between countries. ${ }^{20}$ It is therefore important to identify the current status of PCAS care in Korea in order to make recommendations that are suitable for the country's specific circumstances. The Korean Hypothermia Network (KORHN) investigators previously published retrospective, multicenter, observational studies on postcardiac arrest care quality and outcomes in patients treated with TM between January 2007 and December 2012. ${ }^{21}$ Since then, however, the guidelines have been updated, resulting in changes in TM treatment, and the retrospective data at the time were based on limited research. The KORHN multicenter clinical research consortium for TM in South Korea, founded in 2010, therefore launched a multicenter, prospective registry of OHCA patients, and enrolled approximately 10,000 such patients from 22 participating institutions between October 2015 and December 2018.

Using this database, we aimed to assess the current status of PCAS care in Korea, including TTM and 6-month survival with neurologically favorable outcomes, after adult OHCA patients were treated with TTM.

\section{METHODS}

\section{Study design and participation}

This study was a prospective, multicenter, observational cohort study. Data were collected from the KORHN prospective (KORHNPRO) registry, a web-based registry of OHCA patients treated with TM that aimed to improve postcardiac arrest care quality and outcomes. Adult ( $\geq 18$ years) comatose patients treated with TM between October 2015 and December 2018 were included.

Each investigator, from one of the 22 teaching hospitals that intended to be included in this registry, was required to fill out 
the participation form available on the website of the KORHNPRO registry (http://pro.korhn.or.kr/). A registry manual was created. Investigators were repeatedly educated every 3 months, and researchers were asked to share the queries and others that occurred by attending the education every time. Five clinical research associates monitored the data and improved their quality by sending queries to all investigators, and one data manager eventually examined the data and decided whether records could be accepted or needed revision.

The participating institutions $(n=22)$ were evenly distributed throughout the entire country (Fig. 1). The study design and plan, including the informed consent form, were approved by the institutional review boards of all participating hospitals and registered on the International Clinical Trials Registry Platform (NCT02827422). In accordance with national requirements and the principles of the Declaration of Helsinki, written informed consent was obtained from the patients' legal surrogates rather than from the patients themselves.

\section{Data collection}

The KORHN-PRO registry collected a large amount of information, consisting of 136 variables with 839 datasets.

\section{Baseline patient information}

The date and place of cardiac arrest, hospital visit type (transferred or not), age, sex, height, weight, prearrest Glasgow-Pittsburgh Cerebral Performance Category (CPC)/modified Rankin scale (mRS) score, and comorbidities (coronary heart disease, congestive heart failure, stroke, hypertension, diabetes mellitus, lung disease, renal impairment, liver cirrhosis, and malignancy) were documented.

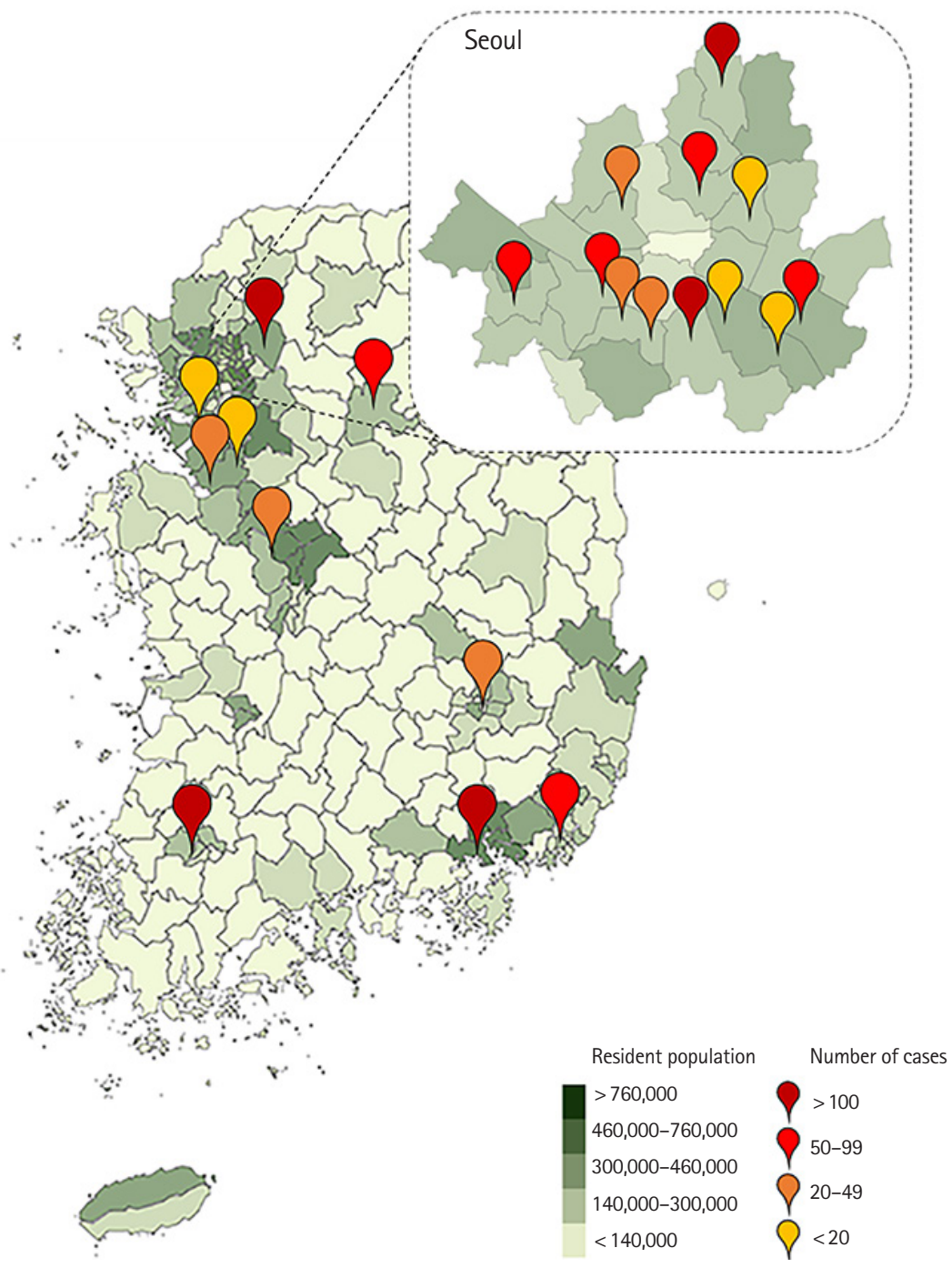

Fig. 1. Geographic distribution of the 22 participating hospitals. 


\section{Resuscitation variables}

Cardiac arrest etiology was dichotomized as medical or nonmedical; medical causes included cases in which the cardiac arrest was presumed to be of cardiac origin or due to other medical causes and those in which there was no obvious cause of cardiac arrest. The initial arrest rhythm was defined as a shockable rhythm when the first monitored rhythm was found to be ventricular fibrillation or pulseless ventricular tachycardia. Other resuscitation variables were as follows: witness of collapse, bystander cardiopulmonary resuscitation (CPR), return of spontaneous circulation (ROSC) status (ROSC after hospital arrival, ROSC before hospital arrival, no ROSC), mechanical chest compression, epinephrine or vasopressin dose, end tidal carbon dioxide concentration during resuscitation, and time from collapse to ROSC.

\section{TTM}

The following TTM data of patients were prospectively collected: target temperature, target duration, temperature monitoring site, hourly body temperature from ROSC to 96 hours, TTM methods (blanket, ice bag, adhesive pad, garment, fan, linen, cold saline, intravascular catheter, lavage, and extracorporeal membrane oxygenation), time from ROSC to start of TM, time from start of TMM to achieving the target temperature, maintenance duration, rewarming duration, controlled normothermia, and duration and maximal temperature after rewarming for 3 days.

\section{In-hospital data including treatments}

Hemodynamic parameters, shock after ROSC (a systolic blood pressure $<90 \mathrm{mmHg}$ for $>30$ minutes, or the need for supportive measures to maintain a blood pressure of $90 \mathrm{mmHg}$ ), the Glasgow Coma Scale score after ROSC, arterial blood gases, 12-lead electrocardiography, and serum troponin levels were measured immediately after ROSC. The lowest hypoxic index on the day, the cardiovascular sequential organ failure assessment score, serum creatinine levels, platelet counts, bilirubin levels, the patient's fluid balance, and their neurologic status (i.e., their Glasgow Coma Scale score, pupillary light reflex, and corneal reflex) were evaluated daily up to 7 days after ROSC. Adverse events within 7 days after ROSC (seizures, bleeding, infections, sepsis, pneumonia, hypokalemia, hypophosphatemia, hypomagnesaemia, hypoglycemia [blood glucose $<60 \mathrm{mg} / \mathrm{dL}$ ], sustained hyperglycemia [blood glucose $>180 \mathrm{mg} / \mathrm{dL}$ for $>4$ hours], tachycardia [ $>130 / \mathrm{min}$ ], and bradycardia [ $<40 / \mathrm{min}]$ ) and rearrest events were documented. The registry contained data on advanced cardiac treatments (e.g., $C A G$, percutaneous coronary intervention, results of $C A G$, coronary artery bypass graft, extracorporeal bypass, intra-aortic balloon pump, use of thrombolytics, electrophysiology study, implant- able cardioverter-defibrillator insertion, and pacemaker insertion), echocardiography, regional wall motion abnormalities, time from ROSC to CAG, and continuous renal replacement therapy.

\section{Outcomes and outcome prediction modality}

The researchers prospectively recorded whether there were any decisions regarding limitations of active treatments during hospital care (i.e., withdrawal of life sustaining therapy, whether patients underwent essential diagnostic testing, or whether do not attempt CPR orders were in place). Neurologic outcomes were investigated by the researchers at each hospital, and assessed at discharge as well as at 1 and 6 months after cardiac arrest. The KORHN protocol recommends a minimalist approach when assigning the Glasgow-Pittsburgh CPC score that is based on four simple questions, and the researchers contacted either surviving discharged patients or their relatives to calculate the score. Follow-up was recommended by face-to-face or telephone interviews.

The various modalities used for outcome prediction were also prospectively collected and included the following: brain computed tomography (CT), brain magnetic resonance imaging, serum neuron-specific enolase every 24 hours for 72 hours, median nerve somatosensory evoked potentials (SSEPs), and conventional electroencephalogram (EEG).

\section{Primary outcome}

The primary outcome was the neurologic outcome assessed using the CPC score 6 months after cardiac arrest according to the recommendations for outcome assessments in comatose cardiac arrest survivors; it was recorded as CPC 1 (good performance), CPC 2 (moderate disability), CPC 3 (severe disability), CPC 4 (vegetative state), or CPC 5 (brain death or death). Neurological outcomes were then dichotomized into good (CPC 1 or 2) or poor (CPC 3 to 5). ${ }^{22,23}$ Additionally, we used the $\mathrm{mRS}$ to assess neurological outcomes, with an mRS score of 0 to 3 being considered a good outcome and an mRS score of 4 to 6 a poor outcome (with an mRS score of 6 indicating death). ${ }^{22,23}$

\section{Statistical analysis}

Categorical variables are presented as frequencies and percentages. Comparisons of categorical variables were performed using chi square tests or Fisher's exact test, as appropriate. We tested the distributions of continuous variables for normality using visual inspection and the Shapiro-Wilk test. Normally distributed data are expressed as means and standard deviations and assessed using Student's t-test. Non-normally distributed data are expressed as medians and interquartile ranges and assessed using the Mann-Whitney U-test. All data were analyzed 
using IBM SPSS version 23.0 (IBM Corp., Armonk, NY, USA).

Significance was set at $\mathrm{P}<0.05$.

Table 1. Demographic and resuscitation data of the patients

\begin{tabular}{lcccr}
\hline & $\begin{array}{c}\text { All } \\
(\mathrm{n}=1,354)\end{array}$ & $\begin{array}{c}\text { Good at } \\
6 \text { months } \\
(\mathrm{n}=413)\end{array}$ & $\begin{array}{c}\text { Poor at } \\
6 \text { months } \\
(\mathrm{n}=941)\end{array}$ & P-value \\
\hline Sex, male & $961(71.0)$ & $322(78.0)$ & $639(67.9)$ & $<0.001$ \\
Age (yr) & $58.0 \pm 15.8$ & $52.7 \pm 14.6$ & $60.3 \pm 15.7$ & $<0.001$ \\
Comorbidity & & & & \\
$\quad$ Healthy & $408(30.1)$ & $171(41.4)$ & $237(25.2)$ & $<0.001$ \\
Coronary artery disease & $269(19.9)$ & $95(23.0)$ & $174(18.5)$ & 0.055 \\
Hypertension & $484(35.7)$ & $120(29.1)$ & $364(38.7)$ & 0.001 \\
Diabetes mellitus & $328(24.2)$ & $64(15.5)$ & $264(28.1)$ & $<0.001$ \\
$\quad$ Cerebrovascular accident & $64(4.7)$ & $17(4.1)$ & $47(5.0)$ & 0.483 \\
Visit type & & & & 0.552 \\
$\quad$ Direct & $943(69.6)$ & $283(68.5)$ & $660(70.1)$ & \\
Transport & $411(30.4)$ & $130(31.5)$ & $281(29.9)$ & \\
Resuscitation variables & & & & \\
Cardiac etiology & $839(62.0)$ & $364(88.1)$ & $475(50.5)$ & $<0.001$ \\
Witnessed & $937(69.2)$ & $347(84.0)$ & $590(62.7)$ & $<0.001$ \\
Bystander CPR & $834(61.6)$ & $281(68.0)$ & $553(58.8)$ & 0.001 \\
Shockable rhythm & $482(35.6)$ & $307(74.3)$ & $175(18.6)$ & $<0.001$ \\
Collapse to ROSC (min) & $30(18-43)$ & $18(13-26)$ & $35(24-47)$ & $<0.001$ \\
Epinephrine (mg) & $2.0(0-4)$ & $0.0(0-1)$ & $2.0(1-4)$ & $<0.001$ \\
Prehospital ROSC & $421(31.1)$ & $283(68.5)$ & $138(14.7)$ & $<0.001$ \\
\hline
\end{tabular}

Values are presented as number (\%), mean \pm standard deviation, or median (interquartile range).

$\mathrm{CPR}$, cardiopulmonary resuscitation; ROSC, return of spontaneous circulation.

\section{RESULTS}

\section{Demographic and baseline characteristics}

During the study period, a total of 10,258 patients with OHCA were initially screened for the registration. Of those, 1,373 patients were treated with TTM and thus included in the KORHNPRO registry. A total of 1,354 patients (98.6\%) with complete data including the 6-month neurologic outcomes were finally included in this study.

The patients' clinical characteristics are shown in Table 1. A total of 961 patients $(71.0 \%)$ were male, and the mean age was 58 years. Patients with a good neurological outcome were significantly more likely to be male and younger $(P<0.001)$. Hypertension was the most frequent comorbidity. Patients with hypertension and diabetes mellitus were more likely to have a poor neurological outcome. There were 839 patients (62.0\%) with presumed cardiac etiology. A total of 937 collapses (69.2\%) were witnessed, and 834 patients (61.6\%) received bystander CPR. Shockable rhythms were identified in 482 patients (35.6\%), and 421 (31.1\%) patients arrived at the ED with prehospital ROSC. Good neurologic outcomes were associated with witness of collapse, bystander CPR, shockable rhythms, shorter times from collapse to ROSC, and prehospital ROSC $(\mathrm{P}<0.001)$.

\section{Clinical findings immediately after ROSC}

Immediately after ROSC, 170 (12.6\%) patients showed ST elevation on 12-lead electrocardiography (Table 2). Patients with a good

Table 2. Clinical findings immediately after return of spontaneous circulation

\begin{tabular}{|c|c|c|c|c|}
\hline & $\begin{array}{c}\text { All } \\
(n=1,354)\end{array}$ & $\begin{array}{c}\text { Good at } \\
6 \text { months }(n=413)\end{array}$ & $\begin{array}{c}\text { Poor at } \\
6 \text { months }(n=941)\end{array}$ & P-value \\
\hline ST-elevation myocardial infarction & $170(12.6)$ & $75(18.2)$ & $95(10.1)$ & $<0.001$ \\
\hline \multicolumn{5}{|l|}{ Neurologic examination } \\
\hline GCS motor & $302(22.3)$ & $201(48.7)$ & $101(10.7)$ & $<0.001$ \\
\hline Pupillary light reflex & $440(32.5)$ & $262(63.4)$ & $178(18.9)$ & $<0.001$ \\
\hline Corneal reflex & $268(19.8)$ & $178(43.1)$ & $90(9.6)$ & $<0.001$ \\
\hline \multicolumn{5}{|l|}{ Hemodynamic status } \\
\hline Mean arterial pressure $(\mathrm{mmHg})$ & $92.2 \pm 31.5$ & $100.9 \pm 30.8$ & $88.2 \pm 30.9$ & $<0.001$ \\
\hline Heart rate (beats/min) & $105.5 \pm 29.2$ & $103.0 \pm 29.3$ & $106.6 \pm 29.0$ & 0.043 \\
\hline Temperature $\left({ }^{\circ} \mathrm{C}\right)$ & $35.7 \pm 1.3$ & $35.9 \pm 1.0$ & $35.6 \pm 1.4$ & 0.001 \\
\hline Pre-induction shock & $695(51.3)$ & $140(33.9)$ & $555(59.0)$ & $<0.001$ \\
\hline Glucose (mg/dL) & $254(189-327)$ & $238(180-294)$ & $266(196-346)$ & $<0.001$ \\
\hline \multicolumn{5}{|l|}{ Arterial blood gas analysis } \\
\hline Hydrogen ion concentration & $7.08(6.92-7.23)$ & $7.22(7.09-7.30)$ & 7.02 (6.89-7.17) & $<0.001$ \\
\hline $\mathrm{PaCO}_{2}(\mathrm{mmHg})$ & $49.0(36.1-71.7)$ & $39.0(32.0-48.2)$ & $56.0(39.2-79.4)$ & $<0.001$ \\
\hline $\mathrm{PaO}_{2}(\mathrm{mmHg})$ & $110.0(72.7-198.0)$ & $108.8(73.9-192.5)$ & $110.0(71.4-199.0)$ & 0.649 \\
\hline Bicarbonate (mmol/L) & $15.0(11.7-18.6)$ & $15.7(12.8-19.1)$ & $14.6(11.3-18.4)$ & $<0.001$ \\
\hline Lactic acid (mmol/L) & $9.7(6.1-12.9)$ & $7.0(4.3-10.9)$ & $10.6(7.4-13.5)$ & $<0.001$ \\
\hline
\end{tabular}

Values are presented as number (\%), mean \pm standard deviation, or median (interquartile range).

$\mathrm{GCS}$, Glasgow Coma Scale; $\mathrm{PaCO}_{2}$, partial pressure of carbon dioxide in arterial blood; $\mathrm{PaO}_{2}$, partial pressure of oxygen in arterial blood. 
neurologic outcome had a significantly higher mean arterial pressure than patients with a poor neurologic outcome. Preinduction shock was more common in patients with a poor neurologic outcome than in those with a good neurologic outcome $(P<0.001)$. The poor neurologic outcome group had higher glucose and elevated lactic acid levels than the good neurologic outcome group $(P<0.001)$.

\section{Neurological outcome and survival according to the first monitored rhythm}

Fig. 2 shows an overview of the registered patients. Of the 1,354 total patients, 550 (40.6\%) survived 6 months, and 413 (30.5\%) had good neurologic outcomes at 6 months. Of the 482 patients who experienced OHCA with shockable rhythms, 337 (69.9\%) survived 6 months, and 307 (63.7\%) had good neurologic outcomes at 6 months.

\section{TTM practices and complications}

The most common target temperature was $33^{\circ} \mathrm{C}$, with an annual increase in the proportion of target temperatures above $35^{\circ} \mathrm{C}$ ( Table 3). The most common target duration was 24 hours, and most patients (89.7\%) were actively rewarmed at a rate of $0.25^{\circ} \mathrm{C} / \mathrm{hr}$ (54.8\%). After rewarming, 736 patients (53.6\%) maintained normothermia for an average of 32.6 hours. The hydrogel pad was most frequently (69\%) used for induction, and 925 (67.4\%) patients used it for maintenance and rewarming (Table 4).
Table 5 shows incidences of complications during TTM. Seizures, bleeding, sepsis, hypoglycemia, sustained hyperglycemia, and rearrest were significantly more frequent in patients with poor neurologic outcomes than in those with good neurologic outcomes.

\section{Cardiac care and circulatory supportive therapy}

Table 6 shows the use of cardiac care after ROSC. CAG was performed in 497 patients (36.7\%), and percutaneous coronary intervention was performed in 206 patients (15.2\%). CAG was per-

Table 3. Targeted temperature management characteristics

\begin{tabular}{|c|c|c|c|c|}
\hline & $\begin{array}{c}2015 \\
(n=61)\end{array}$ & $\begin{array}{c}2016 \\
(n=419)\end{array}$ & $\begin{array}{c}2017 \\
(n=449)\end{array}$ & $\begin{array}{c}2018 \\
(n=425)\end{array}$ \\
\hline \multicolumn{5}{|c|}{ Target temperature $\left({ }^{\circ} \mathrm{C}\right)$} \\
\hline 32 & $0(0.0)$ & $1(0.2)$ & $2(0.4)$ & $0(0.0)$ \\
\hline 33 & $50(82.0)$ & $299(71.4)$ & $335(74.6)$ & $284(66.8)$ \\
\hline 34 & $5(8.2)$ & $24(5.7)$ & $33(7.3)$ & $30(7.1)$ \\
\hline 35 & $4(6.6)$ & $54(12.9)$ & $46(10.2)$ & $55(12.9)$ \\
\hline 36 & $2(3.3)$ & $39(9.3)$ & $33(7.3)$ & $56(13.2)$ \\
\hline 37 & $0(0.0)$ & $2(0.5)$ & $0(0.0)$ & $0(0.0)$ \\
\hline \multicolumn{5}{|c|}{ Target duration (hr) } \\
\hline 12 & $2(3.3)$ & $2(0.5)$ & $0(0.0)$ & $0(0.0)$ \\
\hline 24 & 58 (95.1) & 409 (97.8) & 444 (98.9) & 416 (97.9) \\
\hline 36 & $0(0.0)$ & $1(0.2)$ & $0(0.0)$ & $1(0.2)$ \\
\hline 48 & $1(1.6)$ & $1(0.2)$ & $4(0.9)$ & $5(1.2)$ \\
\hline 60 & $0(0.0)$ & $1(0.2)$ & $0(0.0)$ & $0(0.0)$ \\
\hline 72 & $0(0.0)$ & $4(1.0)$ & $1(0.2)$ & $3(0.7)$ \\
\hline
\end{tabular}

Values are presented as number (\%).

\section{1,354 OHCA treated with TTM}

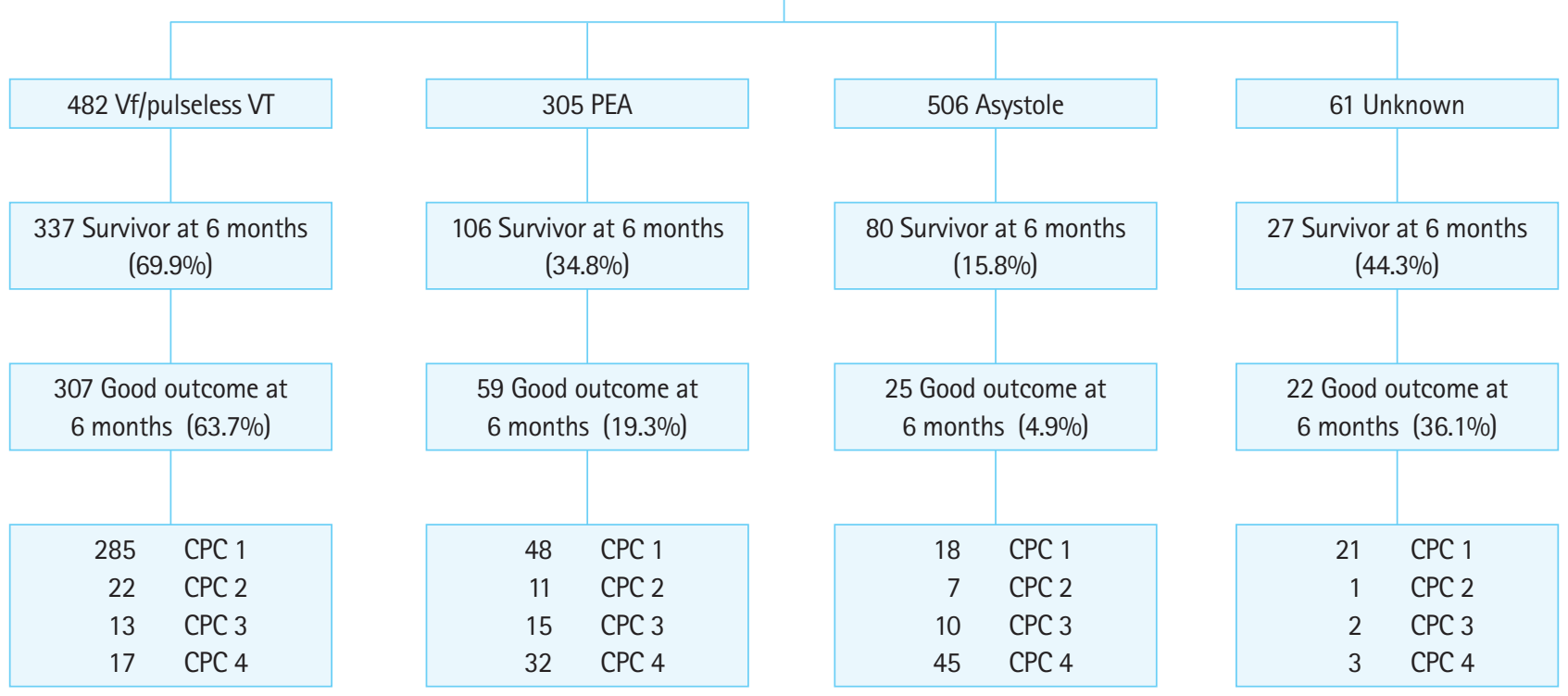

Fig. 2. Survival and neurological outcomes at 6 months of the 1,354 patients with out-of-hospital cardiac arrest (OHCA) treated with targeted temperature management (TTM). Vf, ventricular fibrillation; VT, ventricular tachycardia; PEA, pulseless electrical activity; CPC, Cerebral Performance Category. 
Table 4. Targeted temperature management methods

\begin{tabular}{lcc}
\hline Technique & Induction & $\begin{array}{c}\text { Maintenance to end } \\
\text { of rewarming }\end{array}$ \\
\hline $\begin{array}{l}\text { Feedback device } \\
\text { Hydrogel pad }\end{array}$ & $947(69.0)$ & $925(67.4)$ \\
Blanket or garment & $284(20.7)$ & $268(19.5)$ \\
$\quad$ Endovascular & $136(9.9)$ & $131(9.5)$ \\
Ice pack & $130(9.5)$ & $19(1.4)$ \\
Cold saline & $120(8.7)$ & $5(0.4)$ \\
Cold gastric lavage & $2(0.1)$ & 0 \\
Cold bladder irrigation & $85(6.2)$ & $6(0.4)$ \\
Extracorporeal cooling & $15(1.1)$ & $15(1.1)$ \\
\hline
\end{tabular}

Values are presented as number (\%).

Table 5. Complications during targeted temperature management

\begin{tabular}{lcccr}
\hline & $\begin{array}{c}\text { All } \\
(n=1,354)\end{array}$ & $\begin{array}{c}\text { Good at } \\
6 \text { months } \\
(n=413)\end{array}$ & $\begin{array}{c}\text { Poor at } \\
6 \text { months } \\
(n=941)\end{array}$ & P-value \\
\hline Seizure & $322(23.8)$ & $58(14.0)$ & $264(28.1)$ & $<0.001$ \\
Bleeding & $65(4.8)$ & $8(1.9)$ & $57(6.1)$ & 0.001 \\
Infection & $675(49.9)$ & $196(47.5)$ & $479(50.9)$ & 0.243 \\
$\quad$ Pneumonia & $519(38.3)$ & $175(42.4)$ & $344(36.6)$ & 0.043 \\
$\quad$ Sepsis & $157(11.6)$ & $20(4.8)$ & $137(14.6)$ & $<0.001$ \\
Hypokalemia & $443(32.7)$ & $128(31.0)$ & $315(33.5)$ & 0.370 \\
Hypoglycemia & $152(11.2)$ & $25(6.1)$ & $127(13.5)$ & $<0.001$ \\
Sustained hyperglycemia & $697(51.5)$ & $127(30.8)$ & $570(60.6)$ & $<0.001$ \\
Tachycardia & $273(20.2)$ & $89(21.5)$ & $184(19.6)$ & 0.399 \\
Bradycardia & $96(7.1)$ & $35(8.5)$ & $61(6.5)$ & 0.189 \\
Re-arrest & $260(19.2)$ & $31(7.5)$ & $229(24.3)$ & $<0.001$ \\
\hline
\end{tabular}

Values are presented as number (\%).

formed in $349(72.4 \%)$ of 482 patients with shockable rhythms and in 145 (85.3\%) of 170 patients with ST-elevation myocardial infarction. The median time from ROSC to CAG was 2.7 hours, and 378 (76.1\%) patients underwent CAG within 24 hours.

\section{Modalities for outcome prediction}

Neurologic examinations 72 hours after ROSC were performed in $1,181(87.2 \%)$ patients, and serum neuron-specific enolase was checked in 815 (59.4\%) patients. SSEP was performed in 339 and EEG in 700 patients. Most patients (92.5\%) underwent brain CT examinations, and the median time to exam was 1.5 hours. A total of 655 patients underwent brain magnetic resonance imaging examinations, and the median time to exam was 78 hours for predicting neurologic outcome after OHCA (Table 7).

\section{DISCUSSION}

The KORHN committee launched a multicenter, prospective registry that focuses on OHCA patients treated with TM since Octo-
Table 6. Cardiac care and circulatory supportive therapy

\begin{tabular}{lccc}
\hline & $\begin{array}{c}\text { All } \\
(n=1,354)\end{array}$ & $\begin{array}{c}\text { Shockable } \\
(n=482)\end{array}$ & $\begin{array}{c}\text { STEMI } \\
(n=170)\end{array}$ \\
\hline $\begin{array}{l}\text { Extracorporeal cardiopulmonary } \\
\text { resuscitation }\end{array}$ & $15(1.1)$ & $10(2.1)$ & $6(3.5)$ \\
$\begin{array}{l}\text { Extracorporeal membrane oxygenation } \\
\text { Intraaortic balloon pump }\end{array}$ & $49(3.6)$ & $29(6.0)$ & $20(11.8)$ \\
Coronary angiography & $9(0.7)$ & $5(1.0)$ & $3(1.8)$ \\
$\quad$ Percutaneous coronary intervention & $206(15.2)$ & $156(32.4)$ & $102(60.0)$ \\
Time to coronary angiography (hr) & $2.7(2-21)$ & $2.9(2-79)$ & $2.0(1.0-3.0)$ \\
$\quad$ Early coronary angiography & $378(27.9)$ & $248(51.5)$ & $130(76.5)$ \\
Implantable cardioverter defibrillator & $96(7.1)$ & $90(18.7)$ & $11(6.5)$ \\
Echocardiogram & $723(53.4)$ & $364(75.5)$ & $129(75.9)$ \\
Regional wall motion abnormality & $254(18.8)$ & $154(32.0)$ & $81(47.6)$
\end{tabular}

Values are presented as number (\%) or median (interquartile range). STEMI, ST-elevation myocardial infarction.

Table 7. Modalities for outcome prediction $(n=1,354)$

\begin{tabular}{lc}
\hline Modality & Value \\
\hline Neurological exam at 72 hours & $1,181(87.2)$ \\
Neuron-specific enolase & $815(59.4)$ \\
Electroencephalography & $700(51.8)$ \\
Somatosensory evoked potential & $339(25.0)$ \\
Brain computed tomography & $1,252(92.5)$ \\
Time to exam (hr) & $1.5(1.0-2.6)$ \\
Brain magnetic resonance imaging & $655(48.6)$ \\
Time to exam (hr) & $78(70-91)$ \\
\hline
\end{tabular}

Values are presented as number (\%) or median (interquartile range).

ber 2015. This report briefly describes the characteristics and outcomes of the registry from October 2015 to December 2018. Of the 1,354 registered OHCA survivors treated with TTM, 550 (40.6\%) survived 6 months, and $413(30.5 \%)$ had good neurological outcomes.

This study has several strengths. First, this is the first prospective registry involving multiple centers in Korea. Second, 98.6\% of all enrolled patients provided data on the primary outcome of our study, that is, neurologic outcome at 6 months after cardiac arrest.

The good neurologic outcome rate of all patients was 30.5\%, and the good neurologic outcome rate of patients with initial shockable rhythms was $63.7 \%$. In an earlier TM trial, $46 \%$ of patients with good neurologic outcomes were in the $33^{\circ} \mathrm{C}$ group and $48 \%$ in the $36^{\circ} \mathrm{C}$ group. ${ }^{8}$ However, as this earlier trial did not report rates of good neurologic outcome at 6 months according to initial rhythm, no direct comparison with our registry is possible. In a recently published Time-differentiated therapeutic hypothermia (TH) 48 trial, the rate of good neurologic outcomes at 6 months was 64\% in the 24-hour group and 69\% in the 48-hour group. ${ }^{24}$ 
This means that, in the current study, the proportion of patients with an initial shockable rhythm was lower than the same proportion reported in earlier European trials, such as the just mentioned TTM and TH 48 trials; however, our good outcome rate is similar to those of earlier reports.

In our prospective registry, the most common target temperature was $33^{\circ} \mathrm{C}$, and the most common target duration was 24 hours. Although the optimal target temperature and duration are still unknown, many guidelines recommend a target temperature of $32^{\circ} \mathrm{C}$ to $36^{\circ} \mathrm{C}$ and a minimum target duration of 24 hours. ${ }^{1,5}$ In the United States, the rate of TTM applications has decreased by more than $6 \%$ since the publication of the TTM trial. ${ }^{24}$ The Australian and New Zealand Intensive Care Society reported that the lowest average temperature of PCAS patients in the first 24 hours in the ICU rose after publication of the TM trial, and that this change was associated with an increased frequency of fever and a tendency toward an increase in mortality. ${ }^{25}$ In the Korean prospective registry, although a target temperature of $33^{\circ} \mathrm{C}$ was the most common, the proportion of target temperatures of $33^{\circ} \mathrm{C}$ has been decreasing over the years. However, the current study did not reveal a relationship between the change in target temperature and outcomes. Further research is needed to expand on these findings.

CAG was performed in 497 patients (36.7\%), and emergency $C A G$, which was defined as $C A G$ performed within 24 hours after ROSC, was performed in 378 patients (27.9\%). The median time from ROSC to CAG was 2.7 hours, and 378 (76.1\%) patients underwent CAG within 24 hours. The TH 48 trial reported that emergency CAG was performed in $82.6 \%$ of patients. ${ }^{24}$ Although there was a difference in the initial shockable rhythm rates between the TH 48 trial and the KORHN-PRO registry, our study shows that even for ST-elevation myocardial infarction cases, the emergency CAG rate was 76.5\%; there is thus room for improvement in PCAS care. Additionally, it will be necessary to study the effect of emergency CAG on the Korean population.

Brain CT was the most frequently used modality (92.5\%) for outcome prediction and SSEP the least frequently performed, at a rate of 25\%. According to one survey from the European Society of Intensive Care Medicine, EEG is the most common tool in routine clinical practice, with intermittent EEG, brain $\mathrm{CT}$, and SSEP being considered the most useful tools for assessing prognosis after cardiac arrest. ${ }^{26}$ Future studies using the KORHN-PRO registry data will reveal the associations between outcome predictors and prognosis after cardiac arrest.

Our study has several limitations. First, the KORHN-PRO registry included only patients with OHCA who were treated with TTM. Therefore, we were not able to assess the ratio of TTM applica- tions, the total OHCA survival rate, or the effectiveness of TM. Second, some missing data in the KORHN-PRO registry, in spite of the data manager and clinical research associates monitoring the data and giving feedback to the researchers, might have affected the results. Third, most of the included facilities are teaching or university-affiliated hospitals located within the nation's capital region. Therefore, a selection bias could not be avoided.

In summary, of the 1,354 OHCA survivors treated with TMM, $550(40.6 \%)$ survived 6 months, and $413(30.5 \%)$ had good neurologic outcomes at 6 months. The most common target temperature was $33^{\circ} \mathrm{C}$, and the most common target duration was 24 hours. CAG was performed in 497 patients (36.7\%), and emergency $C A G$, which was defined as CAG performed within 24 hours after ROSC, was performed in 378 patients (27.9\%).

\section{CONFLICT OF INTEREST}

No potential conflict of interest relevant to this article was reported.

\section{REFERENCES}

1. Neumar RW, Shuster M, Callaway CW, et al. Part 1: Executive summary: 2015 American Heart Association guidelines update for cCardiopulmonary resuscitation and emergency cardiovascular care. Circulation 2015;132(18 Suppl 2):S315-67.

2. Perkins GD, Handley AJ, Koster RW, et al. European Resuscitation Council Guidelines for Resuscitation 2015: Section 2. Adult basic life support and automated external defibrillation. Resuscitation 2015;95:81-99.

3. Benjamin EJ, Virani SS, Callaway CW, et al. Heart disease and stroke statistics-2018 update: a report from the American heart association. Circulation 2018;137:e67-492.

4. Peberdy MA, Callaway CW, Neumar RW, et al. Part 9: postcardiac arrest care: 2010 American Heart Association Guidelines for Cardiopulmonary Resuscitation and Emergency Cardiovascular Care. Circulation 2010;122(18 Suppl 3):S768-86.

5. Nolan JP, Soar J, Cariou A, et al. European Resuscitation Council and European Society of Intensive Care Medicine 2015 guidelines for post-resuscitation care. Intensive Care Med 2015;41:2039-56.

6. Hypothermia after Cardiac Arrest Study Group. Mild therapeutic hypothermia to improve the neurologic outcome after cardiac arrest. N Engl J Med 2002;346:549-56.

7. Bernard SA, Gray TW, Buist MD, et al. Treatment of comatose survivors of out-of-hospital cardiac arrest with induced hypothermia. N Engl J Med 2002;346:557-63. 
8. Nielsen N, Wetterslev J, Cronberg T, et al. Targeted temperature management at $33^{\circ} \mathrm{C}$ versus $36^{\circ} \mathrm{C}$ after cardiac arrest. $\mathrm{N}$ Engl J Med 2013;369:2197-206.

9. Sunde $K$, Pytte $M$, Jacobsen $D$, et al. Implementation of a standardised treatment protocol for post resuscitation care after out-of-hospital cardiac arrest. Resuscitation 2007;73:29-39.

10. Chiu YK, Lui CT, Tsui KL. Impact of hypotension after return of spontaneous circulation on survival in patients of out-of-hospital cardiac arrest. Am J Emerg Med 2018;36:79-83.

11. Elmer J, Torres C, Aufderheide TP, et al. Association of early withdrawal of life-sustaining therapy for perceived neurological prognosis with mortality after cardiac arrest. Resuscitation 2016;102:127-35.

12. Gaieski DF, Band RA, Abella BS, et al. Early goal-directed hemodynamic optimization combined with therapeutic hypothermia in comatose survivors of out-of-hospital cardiac arrest. Resuscitation 2009;80:418-24.

13. Johnson NJ, Carlbom DJ, Gaieski DF. Ventilator management and respiratory care after cardiac arrest: oxygenation, ventilation, infection, and injury. Chest 2018;153:1466-77.

14. Khan MS, Shah SM, Mubashir A, et al. Early coronary angiography in patients resuscitated from out of hospital cardiac arrest without ST-segment elevation: a systematic review and meta-analysis. Resuscitation 2017;121:127-34.

15. Kilgannon JH, Jones AE, Shapiro NI, et al. Association between arterial hyperoxia following resuscitation from cardiac arrest and in-hospital mortality. JAMA 2010;303:2165-71.

16. Lee BK, Jeung KW, Lee HY, et al. Association between mean arterial blood gas tension and outcome in cardiac arrest patients treated with therapeutic hypothermia. Am J Emerg Med 2014;32:55-60.

17. McKenzie N, Williams TA, Tohira H, Ho KM, Finn J. A systematic review and meta-analysis of the association between arterial carbon dioxide tension and outcomes after cardiac arrest. Resuscitation 2017;111:116-26.
18. Mulder M, Gibbs HG, Smith SW, et al. Awakening and withdrawal of life-sustaining treatment in cardiac arrest survivors treated with therapeutic hypothermia. Crit Care Med 2014;42: 2493-9.

19. Youn CS, Park KN, Kim SH, et al. The cumulative partial pressure of arterial oxygen is associated with neurological outcomes after cardiac arrest treated with targeted temperature management. Crit Care Med 2018;46:e279-85.

20. Callaway CW, Schmicker R, Kampmeyer $M$, et al. Receiving hospital characteristics associated with survival after out-ofhospital cardiac arrest. Resuscitation 2010;81:524-9.

21. Lee BK, Park KN, Kang GH, et al. Outcome and current status of therapeutic hypothermia after out-of-hospital cardiac arrest in Korea using data from the Korea Hypothermia Network registry. Clin Exp Emerg Med 2014;1:19-27.

22. Rittenberger JC, Raina K, Holm MB, Kim YJ, Callaway CW. Association between Cerebral Performance Category, Modified Rankin Scale, and discharge disposition after cardiac arrest. Resuscitation 2011;82:1036-40.

23. Haywood K, Whitehead L, Nadkarni VM, et al. COSCA (Core Outcome Set for Cardiac Arrest) in adults: an advisory statement from the international liaison committee on resuscitation. Resuscitation 2018;127:147-63.

24. Kirkegaard $H$, Soreide $E$, de Haas I, et al. Targeted temperature management for 48 vs 24 hours and neurologic outcome after out-of-hospital cardiac arrest: a randomized clinical trial. JAMA 2017;318:341-50.

25. Salter $R$, Bailey $M$, Bellomo $R$, et al. Changes in temperature management of cardiac arrest patients following publication of the target temperature management trial. Crit Care Med 2018;46:1722-30.

26. Friberg H, Cronberg T, Dunser MW, Duranteau J, Horn J, Oddo M. Survey on current practices for neurological prognostication after cardiac arrest. Resuscitation 2015;90:158-62. 